\title{
Star formation in AGNs at the hundred parsec scale using MIR high-resolution images
}

\author{
Daniel Ruschel-Dutra, ${ }^{1,2 \star}$ José Miguel Rodríguez Espinosa, ${ }^{1}$ \\ Omaira González Martín, ${ }^{1,3,4}$ Miriani Pastoriza $^{2}$ and Rogério Riffel ${ }^{2}$ \\ ${ }^{1}$ Instituto de Astrofísica de Canarias, E-38205, La Laguna, Spain \\ ${ }^{2}$ Departamento de Astronomia, Instituto de Física da Universidade Federal do Rio Grande do Sul, 91501-970, Porto Alegre, Brazil \\ ${ }^{3}$ Departamento de Astrofísica, Universidad de La Laguna (ULL), E-38205 La Laguna, Spain \\ ${ }^{4}$ Centro de Radioastronomía Astrofísica (CRyA-UNAM), 3-72 (Xangari), 8701 Morelia, Mexico
}

Accepted 2016 December 14. Received 2016 December 14; in original form 2014 November 30

\begin{abstract}
It has been well established in the past decades that the central black hole masses of galaxies correlate with dynamical properties of their harbouring bulges. This notion begs the question of whether there are causal connections between the active galactic nucleus (AGN) and its immediate vicinity in the host galaxy. In this paper, we analyse the presence of circumnuclear star formation in a sample of 15 AGN using mid-infrared observations. The data consist of a set of $11.3 \mu \mathrm{m}$ polycyclic aromatic hydrocarbon emission and reference continuum images, taken with ground-based telescopes, with sub-arcsecond resolution. By comparing our star formation estimates with AGN accretion rates, derived from X-ray luminosities, we investigate the validity of theoretical predictions for the AGN-starburst connection. Our main results are: (i) circumnuclear star formation is found, at distances as low as tens of parsecs from the nucleus, in nearly half of our sample (7/15); (ii) star formation luminosities are correlated with the bolometric luminosity of the $\operatorname{AGN}\left(L_{\mathrm{AGN}}\right)$ only for objects with $L_{\mathrm{AGN}} \geq 10^{42} \mathrm{erg} \mathrm{s}^{-1}$; (iii) low-luminosity AGNs $\left(L_{\mathrm{AGN}}<10^{42} \mathrm{erg} \mathrm{s}^{-1}\right)$ seem to have starburst luminosities far greater than their bolometric luminosities.
\end{abstract}

Key words: galaxies: active-galaxies: evolution-galaxies: nuclei.

\section{INTRODUCTION}

The properties of the bulge of galaxies correlate with the properties of their supermassive black holes (SMBH; Kormendy \& Richstone 1995, and references therein). Arguably, the most widely known among these is the correspondence between the mass $\left(M_{\bullet}\right)$ of the SMBH and the velocity dispersion of stars in the bulge $(\sigma)$, in short the $M_{\bullet}-\sigma$ relation (Ferrarese \& Merritt 2000; Gebhardt et al. 2000; Gültekin et al. 2009; McConnell \& Ma 2013). Relationships such as these raise the question of whether or not there is a causal connection between the observed properties of the active nucleus and the host galaxy.

One of the possible physical links between the accretion-powered nuclear activity and its immediate vicinity is the circumnuclear ${ }^{1}$ star formation, as this implies a common mechanism for fuelling the growth of both the SMBH and the stellar bulge. Early works on the subject have shown the ubiquitous presence of star formation

\footnotetext{
^E-mail: daniel.ruschel@ufrgs.br

${ }^{1}$ Here and throughout this paper, circumnuclear refers to scales of the order of hundreds of parsecs or less.
}

in Seyfert galaxies (Rodriguez Espinosa, Rudy \& Jones 1987; González-Delgado \& Pérez 1993; Cid Fernandes et al. 2001; Kauffmann et al. 2003; Davies et al. 2007; Riffel et al. 2007). There have also been a number of articles suggesting that the feedback from accretion on to the SMBH would quench star formation by heating the available gas, deterring the gravitational collapse of molecular clouds (Silk \& Rees 1998; Vollmer \& Davies 2013, and references therein). On the other hand, the turbulence generated by supernova explosions could be responsible for the loss of angular momentum that ultimately leads the gas to the accretion disc (Kawakatu \& Wada 2008; Hopkins \& Quataert 2010; Wutschik, Schleicher \& Palmer 2013).

The mid-infrared (MIR) emission from polycyclic aromatic hydrocarbon $(\mathrm{PAH})$ molecules has frequently been used as a tracer of star formation (e.g. Tielens 2008; Wu et al. 2009; Diamond-Stanic \& Rieke 2010; Gallimore et al. 2010; Ruschel-Dutra et al. 2014). These emission bands are produced when UV photons from young stars heat molecules to temperatures of the order of $1000 \mathrm{~K}$. The energy is subsequently radiated by fluorescence through the many molecular modes of oscillation and vibration. Since it is unlikely that such large molecular species would survive the intense radiation field from the active galactic nuclei (AGNs, Voit 1992; 
Siebenmorgen, Krügel \& Spoon 2004), the analysis of their emission is virtually free from the degeneracy considerations that are relevant for ionic lines. However, it has been argued that the $11.3 \mu \mathrm{m}$ $\mathrm{PAH}$ band is an indicator of recent star formation $\left(\sim 10^{8}\right.$ yr DíazSantos et al. 2010).

This study aims at investigating the relationship between the AGN activity and the recent star formation in its vicinity. It is only natural to search for a causal relation between these phenomena at the smallest possible scales, e.g. at radii of tens of parsecs from the central engine. Such regions are often enshrouded in dusty clouds, favouring observations in wavelengths other than the optical (Nenkova et al. 2008; Ramos Almeida et al. 2011; Sales et al. 2011; González-Martín et al. 2013). At present, ground-based MIR observations with 10 -m class telescopes offer the possibility of obtaining high-resolution images $(\sim 0.4 \operatorname{arcsec})$ at the same time avoiding most of the extinction from the interstellar environment. Therefore, here we analyse high spatial resolution PAH emission images from two similar ground-based instruments, namely the Very Large Telescope (VLT) Imager and Spectrometer for mid-Infrared (VISIR) and the CanariCam attached to the Gran Telescopio Canarias (GTC).

This paper is structured as follows: in Section 2, we discuss the sample selection, observation strategy and reduction process; our estimates for star formation rates (SFRs), as well as the morphological features of the star-forming regions are examined in Section 3; Section 4 examines how the empirical data compare with numerical models for the AGN-SB relation; Section 5 contains a discussion of the results, and finally in Section 6, we present our conclusions.

\section{THE DATA}

\subsection{Sample selection}

The target set for this study began with the selection of four galaxies, three of which were known to harbour AGNs, for observation with GTC/CanariCam, following a criteria of spectral classification diversity. The chosen sources were the low-ionization nuclear emission-line region (LINER) NGC 2146, the Seyfert 1 NGC 931 and the Seyfert 2's NGC 1194 and NGC 2273, the former being classified as a Compton-thick source based on X-ray data (Guainazzi et al. 2005). The selected sources also followed a technical limit of detectability for a reasonable integration time of roughly $0.2 \mathrm{Jy}$ in the $12-\mu \mathrm{m}$ filter of IRAS. This is also the lower limit in the extended $12 \mu \mathrm{m}$ galaxy sample (Rush, Malkan \& Spinoglio 1993), although NGC 2273 violates the $|b|>25^{\circ}$ restriction of this catalogue. Lastly, only galaxies with projected spatial resolutions of the order of hundreds of parsecs were considered.

In order to add statistical weight to this study, 12 sources observed with VISIR at the VLT were added. The data for these targets were taken from the atlas published by Asmus et al. (2014). These additional galaxies consist of all the targets that are clearly detected in both the PAH2 and Si-5 filters and respect the parameters of the last paragraph. The similarity between the filters in CanariCam and VISIR allows for a consistent combined analysis. The spatial scale limit applied to the proprietary data is also respected by the VISIR observations. Basic parameters for the complete sample of 15 AGNs are shown in Table 1.

Our sample of nearby AGN is far from being complete both in volume and luminosity. A cross-match between the AGN catalogue of Véron-Cetty \& Véron (2010) and the extended 12- $\mu \mathrm{m}$ galaxy sample (Rush et al. 1993) returns 44 sources with $z<0.03$ and spectral classification of either Seyfert or LINER. If we extrapolate on the region close to the galactic equator left out from Rush et al. (1993), there should be $\sim 76$ galaxies that fit the description. Consequently, our sample represents close to 20 per cent of the AGNs with $z<0.03$ and $F_{12}>0.2 \mathrm{Jy}$.

\subsection{Observations and data reduction}

New proprietary data were acquired with CanariCam in the filters PAH2 $\left(\lambda_{\mathrm{c}}=11.26 \mu \mathrm{m}\right)$ and $\operatorname{Si} 5\left(\lambda_{\mathrm{c}}=11.53 \mu \mathrm{m}\right)$. Additional archival data consist of VISIR observations with filters PAH2 $\left(\lambda_{\mathrm{c}}=11.26 \mu \mathrm{m}\right)$ and PAH2 $\_2\left(\lambda_{\mathrm{c}}=11.73 \mu \mathrm{m}\right)$. This set of four filters, a pair for each instrument, was chosen to yield the closest possible to continuum-free PAH emission images. The transmission profiles of all the filters employed in this work are shown in Fig. 1, where the transmission coefficient was normalized so that the total area under each curve equals unity.

The field of view for CanariCam is a rectangle measuring 26 $\operatorname{arcsec} \times 19 \operatorname{arcsec}$, with a spatial sampling of 0.08 arcsec pixel ${ }^{-1}$. For VISIR, however, we used data from two distinct observation modes, with square fields of view of sides $32.5 \operatorname{arcsec}$ and

Table 1. Observation log.

\begin{tabular}{|c|c|c|c|c|c|c|c|c|}
\hline \multirow[b]{2}{*}{ Target } & \multirow[b]{2}{*}{ RA (J2000) } & \multirow[b]{2}{*}{ DEC (J2000) } & \multirow[b]{2}{*}{ Sp. type } & \multirow[b]{2}{*}{ Instrument } & \multicolumn{2}{|c|}{ PAH 11.3} & \multicolumn{2}{|c|}{ Si5 11.6} \\
\hline & & & & & Date obs. & $t_{\exp }(\mathrm{s})$ & Date obs. & $t_{\exp }(\mathrm{s})$ \\
\hline ESO 005-G004 & $06^{\mathrm{h}} 05^{\mathrm{m}} 41^{\mathrm{s}} .7$ & $-86^{\circ} 37^{\prime} 55^{\prime \prime} 0$ & Sy2 & VISIR & $2010-11-22$ & 900 & $2010-11-22$ & 900 \\
\hline ESO 383-G035 & $13^{\mathrm{h}} 35^{\mathrm{m}} 53^{\mathrm{s}} .8$ & $-34^{\circ} 17^{\prime} 43^{\prime \prime} .8$ & Sy1.2 & VISIR & 2004-04-14 & 180 & $2010-03-10$ & 360 \\
\hline IC 4329A & $13^{\mathrm{h}} 49^{\mathrm{m}} 19^{\mathrm{s}} \cdot 2$ & $-30^{\circ} 18^{\prime} 33^{\prime \prime} .8$ & Sy 1.2 & VISIR & 2010-03-12 & 180 & 2009-05-10 & 60 \\
\hline IC 5063 & $20^{\mathrm{h}} 52^{\mathrm{m}} 02^{\mathrm{s}} .3$ & $-57^{\circ} 04^{\prime} 07^{\prime \prime} .6$ & Sy2 & VISIR & 2006-05-05 & 180 & $2005-06-10$ & 200 \\
\hline Mrk 1239 & $09^{\mathrm{h}} 52^{\mathrm{m}} 19^{\mathrm{s}} .1$ & $-01^{\circ} 36^{\prime} 43^{\prime \prime} .5$ & Sy1.5 & VISIR & 2005-01-28 & 1000 & 2006-03-12 & 600 \\
\hline NGC 931 & $02^{\mathrm{h}} 28^{\mathrm{m}} 14{ }^{\mathrm{s}} .4$ & $+31^{\circ} 18^{\prime} 41^{\prime \prime} .4$ & Sy1.0 & CanariCam & 2013-09-05 & 278 & 2013-09-05 & 265 \\
\hline NGC 1194 & $03^{\mathrm{h}} 03^{\mathrm{m}} 49^{\mathrm{s}} .1$ & $-01^{\circ} 06^{\prime} 13^{\prime \prime} .0$ & Sy2 & CanariCam & 2013-09-03 & 625 & 2013-09-04 & 199 \\
\hline NGC 2146 & $06^{\mathrm{h}} 18^{\mathrm{m}} 37^{\mathrm{s}} .7$ & $+78^{\circ} 21^{\prime} 25^{\prime \prime} .3$ & LINER & CanariCam & 2013-09-04 & 139 & 2013-09-03 & 662 \\
\hline NGC 2273 & $06^{\mathrm{h}} 50^{\mathrm{m}} 08^{\mathrm{s}} .6$ & $+60^{\circ} 50^{\prime} 44^{\prime \prime} .5$ & Sy2ct & CanariCam & 2013-09-08 & 625 & 2013-09-06 & 662 \\
\hline NGC 5128 & $13^{\mathrm{h}} 25^{\mathrm{m}} 27^{\mathrm{s}} .6$ & $-43^{\circ} 01^{\prime} 08^{\prime \prime} .8$ & Sy2 & VISIR & 2006-04-09 & 180 & 2006-03-15 & 600 \\
\hline NGC 5506 & $14^{\mathrm{h}} 13^{\mathrm{m}} 14.9$ & $-03^{\circ} 12^{\prime} 27^{\prime \prime} .2$ & Sy1.9 & VISIR & $2010-02-23$ & 180 & 2006-06-06 & 600 \\
\hline
\end{tabular}




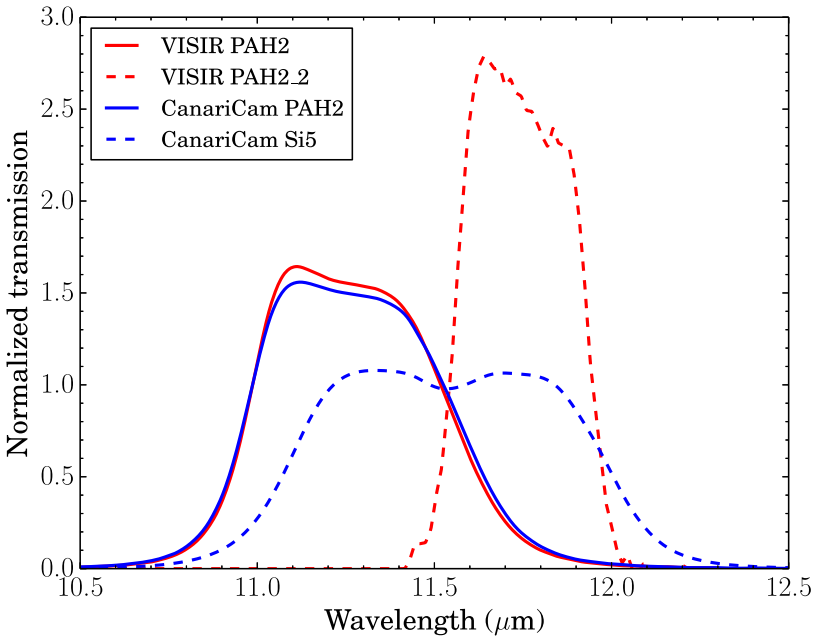

Figure 1. Transmission profiles of the VISIR (red) and CanariCam (blue) filters. Solid and dashed lines represent the emission and continuum filters, respectively, with respect to the rest-frame wavelength. The transmission integral of each filter has been normalized.

$19.2 \mathrm{arcsec}$. The corresponding pixel scales are $0.127 \mathrm{arcsec} \mathrm{pixel}^{-1}$ and 0.075 arcsec pixel ${ }^{-1}$.

The CanariCam data acquisition followed the standard recipe for MIR ground-based observations, with thermal emission from the telescope and the atmosphere being removed by the chopping/nodding technique. The same applies to the archival VISIR data. In the latter case, some of the nod frames that had intense detector artefacts were classified by visual inspection and discarded, resulting in a total exposure time slightly lower than the one originally intended.

The reduction process for the CanariCam data employed the REDCAN pipeline (González-Martín et al. 2013), with the addition of a recently developed algorithm to register the centroid in a sequence of nod images and realign the exposures. This allowed us to obtain nearly diffraction limited images from what was otherwise a seeing limited stack. Standard stars, observed not more than an hour apart from the science exposures, were used to flux calibrate the images. The VISIR images were used as published in Asmus et al. (2014), thus we refer the reader to that paper for details about the reduction process.

\section{IMAGING ANALYSIS}

In this section, we discuss the methodology and results from the analysis of the MIR images. At first the problem of isolating the location of PAH emission is investigated, followed by a description of the new observations with CanariCam. Finally, photometric measurements are discussed.

\subsection{PAH emission maps}

The simplest approach to the problem of isolating the $11.3 \mu \mathrm{m}$ PAH emission would be to produce maps, employing a method to compensate for the different continuum levels in both filters. The objective here is not yet to reach an accurate measurement of PAH emission but rather to locate excesses in the PAH sampling filter with respect to the expected continuum level.

The width of MIR filters tends to be considerably larger than their counterparts in the optical range, close to half a micrometre, as can be seen in Fig. 1. With central wavelengths separated by distances that may be smaller than the filter's full width at halfmaximum (FWHM), it is clear that there is considerable overlap between the emission and reference filters in both instruments. This is particularly true in the case of CanariCam, where the continuum reference filter has nearly 60 per cent of its transmission curve in common with the PAH2 filter. Furthermore, the distance between the filters, although small in comparison to their FWHM, is large enough for differences in continuum levels to become noticeable. Therefore, a simple subtraction of the continuum level, as probed by the reference filter, cannot lead to an accurate estimate of the PAH emission.

Analysing tens of AGNs with already published high-resolution MIR spectra (e.g. González-Martín et al. 2013; Esquej et al. 2014; Ruschel-Dutra et al. 2014; Sales et al. 2014), one finds that lowresolution spectra, taken with the infrared spectrometer (IRS) aboard the Spitzer Space Telescope, show an underlying continuum that is very similar to that of the high-resolution spectra. The reason for this agreement lies in the dominant role of power law and warm dust emission associated with the AGN, plus the silicate absorption bands at $9.7 \mu \mathrm{m}$ and $16.8 \mu \mathrm{m}$. Although there are cases in which these absorption bands are not directly linked to the active nucleus, they still affect the same effect on the emerging nuclear spectrum.

In order to produce a model of the continuum, we examined the Spitzer/IRS spectra of our targets, available at the Spitzer archive, ${ }^{2}$ with the spectral analysis tool PAHFIT (Smith et al. 2007). By fitting the spectrum as a combination of continuum emission, silicate absorption and emission from molecules and ions, the code is able to return a sophisticated estimate for the continuum shape. Since we are studying galaxies, the redshift of the spectrum had to be taken into consideration when producing the model. The reader should keep in mind that Spitzer/IRS has a slit width of 3.6 arcsec and therefore is sampling a much larger region of the galaxy than ground-based observations. Nevertheless, the dominance of the nucleus over the host galaxy makes it possible to obtain a fair approximation of the continuum using low-resolution spectroscopy.

We use this continuum emission as an estimate of the slope of the continuum under the PAH feature. Assuming that the same continuum shape holds throughout the image's field of view, we can then produce a qualitative map of the deviation from the null hypothesis, which is having no PAH emission at all. In other words, we are building a qualitative map of PAH 'excess'. The operation can be described by the equation

$E=\left|I_{1}-I_{2} \frac{\int F_{1} C \mathrm{~d} \lambda}{\int F_{2} C \mathrm{~d} \lambda}\right|$,

where $E$ is the difference from the null hypothesis, $I_{n}$ is the image in the $n$ filter, $F_{n}$ is the normalized transmission function of the $n$ filter and $C$ is the function describing the semi-empirical continuum. This model is limited by the possibility of spatial variations of the continuum function, which could also produce an 'excess' as the one described above. Nevertheless, there is currently no better alternative to infer the continuum slope. We would also like to emphasize that the continuum function is independently modelled for each target according to its Spitzer/IRS spectrum and redshift.

Employing this method, we conclude that from the 15 galaxies in our sample only four show PAH emission in the image subtraction. Of these, only NGC 253 displays an extended structure, with all the

\footnotetext{
${ }^{2}$ http://sha.ipac.caltech.edu/applications/Spitzer/SHA/
} 


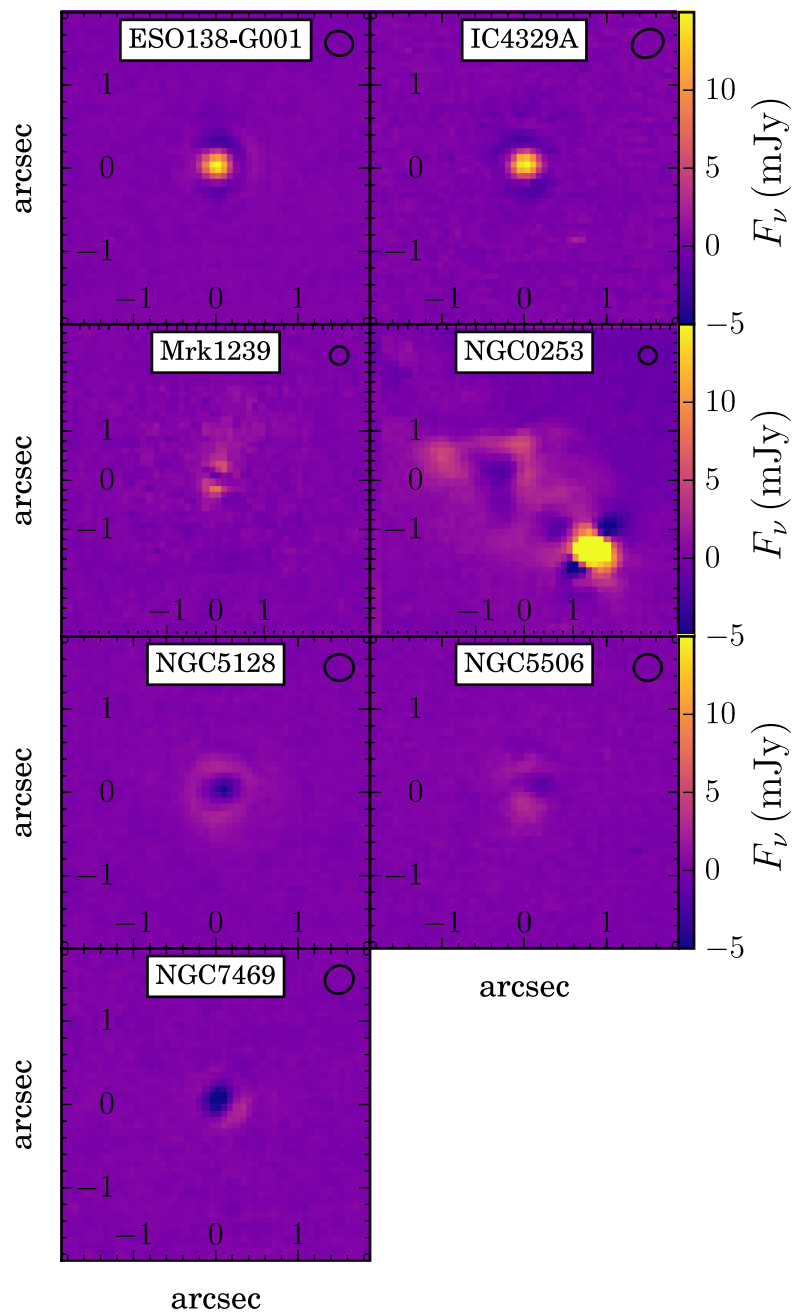

Figure 2. Continuum subtracted $11.3 \mu \mathrm{m}$ PAH images for the galaxies classified as displaying resolved or unresolved emission. The ellipses in the upper-right corner of each image represent the FWHM of the standard star.

other galaxies appearing as unresolved sources. The PAH emission maps that resulted from the continuum subtraction are displayed in Fig. 2 for the spatially unresolved and resolved sources. Notably, as can be seen in Table 1, the images on each filter have not been taken in the same observing night for all the targets. Therefore, the point spread function (PSF) of the images is not naturally matched. Perhaps the most notable case of PSF mismatch is the observations of Mrk 1239, which show lobes extending beyond the FWHM of the central source. We thus refrain from assertions on morphology features that are below the wider of the two PSFs. Considering this caveat, only NGC 253 can be safely classified as having extended PAH emission.

Apart from the four galaxies with larger fluxes on the PAH images, we find two sources, namely NGC 5128 and NGC 7469, which have fluxes in the PAH images lower than the expected from the inferred continuum slope. This effect can be due to an overestimate of this slope or to some of the emission from the molecular band 'leaking' into the reference filter. This last issue is further discussed in Section 4 and in the appendix, where we present our method for dealing with the effects of redshift in the relative fluxes between the filters. Additionally, NGC 5506 shows a small, almost unresolved, $\mathrm{PAH}$ 'excess' and a region where the continuum image is more

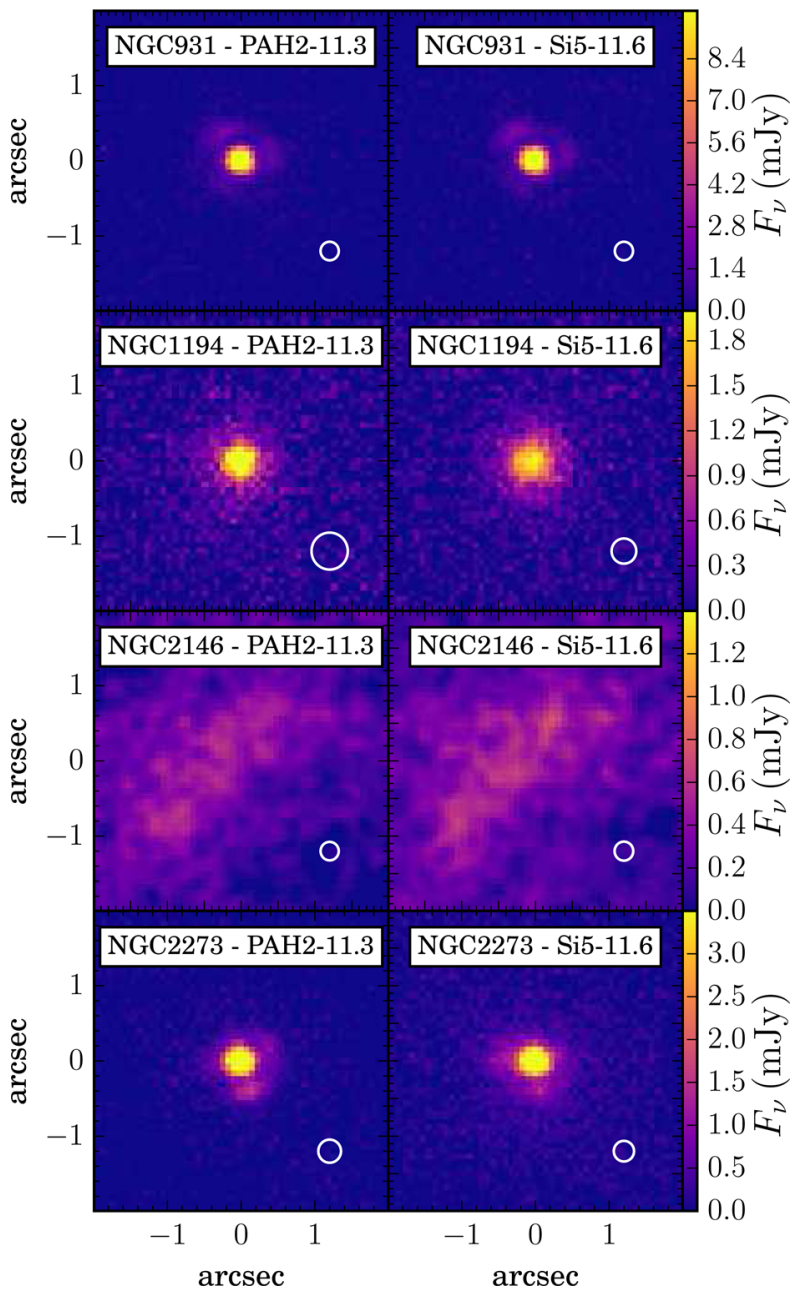

Figure 3. Images acquired with CanariCam. With the exception of NGC 2146 , for which no clear nucleus can be identified, all the other galaxies in this set appear as point-like sources. In order to highlight the extended structure of NGC 2146, its image has been convoluted by a Gaussian. The faint ring-like structures seen in NGC 931 and NGC 2273 are very likely instrumental artefacts (see text). In all figures north is up, east is left, and the ellipse in the lower right represents the FWHM of the standard star.

intense than expected, leading to slightly negative values in Fig. 2. Since the net result is close to zero, we conservatively exclude this galaxy from the list of positive detections.

It is important to keep in mind the projected scale of these images and consequently the maximum radius of an unresolved source. For Mrk 1239, the farthest galaxy in Fig. 2, the PAH emitting region is at most 400 pc away from the central engine, and for the nearest target, namely NGC 253, the same region is no further than $19 \mathrm{pc}$. In the case of NGC 253, structures seen in emission are coincidental with $\mathrm{H}$ II regions already identified in the literature (Forbes et al. 2000; Lira et al. 2007).

\subsection{Proprietary CanariCam images}

Since some of our targets are having their MIR images published for the first time in this paper, we take this opportunity to examine their images in more depth. Four galaxies were observed with CanariCam: NGC 931, NGC 1194, NGC 2146 and NGC 2273. The images in the filters PAH2 - 11.3 $\mu \mathrm{m}$ and $\mathrm{Si} 5-11.6 \mu \mathrm{m}$ for the four galaxies are shown in Fig. 3. For display purposes, the images 
of NGC 2146 have been convolved with a Gaussian with $\sigma$ of one pixel, in order to emphasize the large-scale structure.

At this depth and spatial resolution, the galaxies that harbour an AGN appear as point-like sources in both filters, including the relatively close NGC $2273(z=0.006)$. In contrast, NGC 2146 shows diffuse emission in the form of a band extending from southeast to north-west. The direction of this structure coincides with the dense dust lane identifiable in optical and near-infrared images of this galaxy (e.g. Martini et al. 2003). Although this galaxy is classified as a LINER, no clear nucleus could be identified, and therefore it was left out of the AGN sample. We conclude that this galaxy probably has a LINER-like emission attributable to sources other than the SMBH accretion disc, such as shocks or evolved stars (Filippenko \& Halpern 1984; Stasińska et al. 2008).

Only one of these four sources shows an appreciable difference in flux between filters, namely the Seyfert 2 (Sy2) NGC 1194. The similarity in filter fluxes is an expected result, since the transmission curves overlap and the distance between central wavelengths is comparable to the FWHM of the emission band we are trying to probe.

\subsection{Artefacts in CanariCam imaging}

In some of the images, particularly in the case of NGC 931 and NGC 2273 , one can clearly see a pattern of three bright spots circling the central object. Since the same pattern is also visible in the standard star images, they are almost certainly not real. This pattern is a combination of several effects: the hexagonal shape of GTC's mirror and its segments; a small difference in phase between segments and small guiding errors. The effect is not apparent in the images of NGC 1194 due to its lower signal-to-noise ratio.

The last of the above-mentioned issues is related to the telescope guiding and the way CanariCam records the data. Each saveset is the result of nearly $6 \mathrm{~s}$ of chop cycles, which are stored in a buffer before being written as a file. The source position between frames varies as much as $\sim 0.8$ arcsec peak to peak, and in fact it is apparent by the structures seen in each of the savesets that such variations also occur between chop cycles. Registered stacking of the accumulated chop frames can easily solve the problem of image movement between savesets, as long as the targets are bright enough to be detected in each frame. Nevertheless, movement within each saveset requires a guiding correction frequency at least equal to the chopping frequency.

Unlike the diffraction features, which are directly related to the relative position between the detector and the primary mirror, the guiding artefacts are stochastic in nature. Thus, it is impossible to eliminate them by simple comparison with the standard star. These effects, while potentially harmful to the morphological analysis, are in no way detrimental to the photometry, provided that the apertures include the stray light.

\subsection{Photometry}

Fluxes were obtained from the images through aperture photometry, considering virtual pupils with radii equal to the FWHM of the standard star employed in the flux calibration. The background levels were evaluated from an annulus with width equal to the aperture's radius, separated from the latter by half its radius. All the sums were performed by our own routines which include treatment of partial pixels. Photometric data are displayed in Table 2.

Signal-to-noise ratio estimates for MIR images have a few differences from their optical counterparts. The consideration that atmospheric emission follows a Poisson distribution does not hold for a chop frequency of a few tens of milliseconds. The reason is that at such short intervals there is a significant correlation between background levels in subsequent frames. Moreover, the background level is also a function of the emission from the telescope itself, making it even more time dependent. We chose to estimate the noise levels from the standard deviation of the background in the annulus.

In Figs 4 and 5, we present the photometric points for the 15 galaxies in the sample, plotted along archival spectra from Spitzer/IRS. Since the spatial resolution of Spitzer is much lower than that of the data of CanariCam and VISIR, the flux in the nuclear

Table 2. Photometric fluxes.

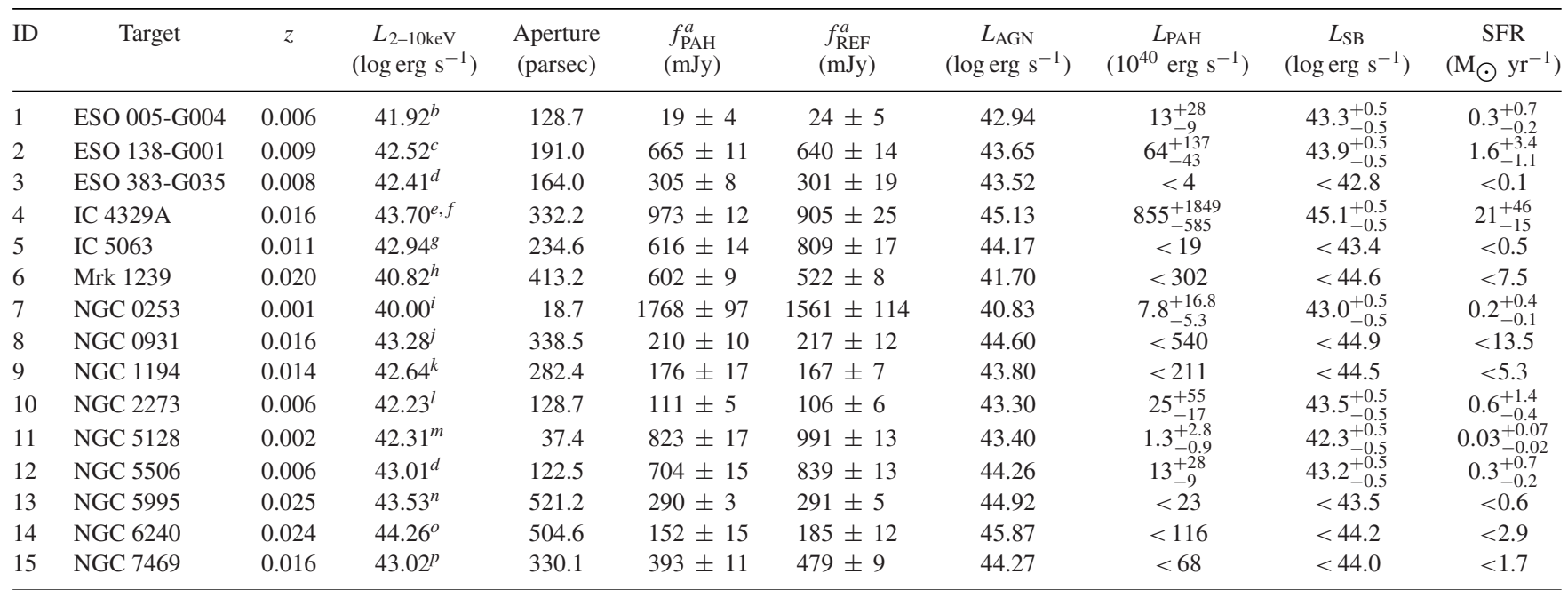

Notes. ${ }^{a}$ These flux densities are the direct result of aperture photometry over the monochromatic images, without any of the procedures for continuum subtraction or redshift compensation discussed in the text. References for the X-ray luminosities: ${ }^{b}$ Winter et al. (2009); ${ }^{c}$ Piconcelli et al. (2011); ${ }^{d}$ Nandra et al. (2007); ${ }^{e}$ Shinozaki et al. (2006); ${ }^{f}$ Bianchi et al. (2009); ${ }^{g}$ Marinucci et al. (2012); ${ }^{h}$ Corral et al. (2011); ${ }^{i}$ Müller-Sánchez et al. (2010); ${ }^{j}$ Ueda et al. (2011); ${ }^{k}$ Greenhill, Tilak \& Madejski (2008); ${ }^{l}$ Awaki et al. (2009); ${ }^{m}$ Shu, Yaqoob \& Wang (2011); ${ }^{n}$ Ueda et al. (2005); ${ }^{o}$ González-Martín et al. (2006); ${ }^{p}$ González-Martín \& Vaughan (2012). 


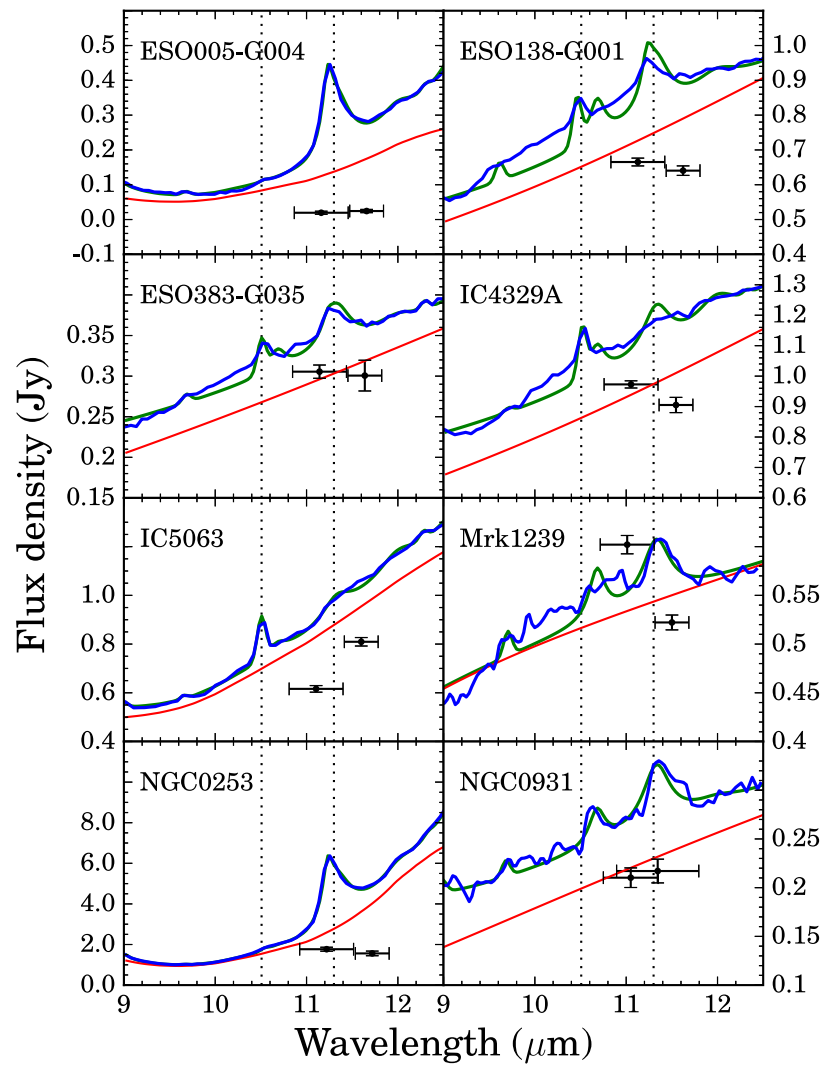

Figure 4. Spectra from Spitzer/IRS for the first eight galaxies in the sample. Blue lines are the observed spectra shifted to the rest frame; red lines represent the continuum emission with the silicate absorption as considered by PAHFIT; vertical dotted lines mark the wavelength of the $10.5 \mu \mathrm{m}$ [SIV] line and the $11.3 \mu \mathrm{m}$ PAH band; green lines represent the best theoretical match to the spectrum. The dots are the photometric flux points shifted to the central wavelength they are sampling at the given redshift, with error bars representing the filter's FWHM and uncertainties in the flux measurements, in the horizontal and vertical directions, respectively.

extractions of the later is naturally smaller. We also show in these figures the continuum inferred from the spectral fitting with PAHFIT.

The photometry of all the other galaxies seems to be in good agreement with the Spitzer/IRS spectra, in the sense that the photometric points are at most equal to the spectroscopic flux. Interestingly, galaxies showing prominent $10.5 \mu \mathrm{m}$ [SIV] emission are the ones that show better agreement between nuclear photometry and host galaxy spectrum, probably due to the prevalence of the central source. Whereas in galaxies with weak [SIV] emission, the AGN is correspondingly less dominant in the low-spatial-resolution spectrum. Targets showing very strong PAH emission in the Spitzer/IRS spectra tend to have nuclear fluxes well below those of the host galaxy and even below PAHFIT's estimate for the continuum.

\section{STAR FORMATION RATES}

In order to estimate the circumnuclear SFRs based on the MIR photometry, we developed a method based on the difference between the photometric fluxes already discussed and careful measurements of the continuum slope. We stress that given the different redshifts of the galaxies and the large variations in silicate profile around $10 \mu \mathrm{m}$, it is impossible to determine the flux in PAH emission

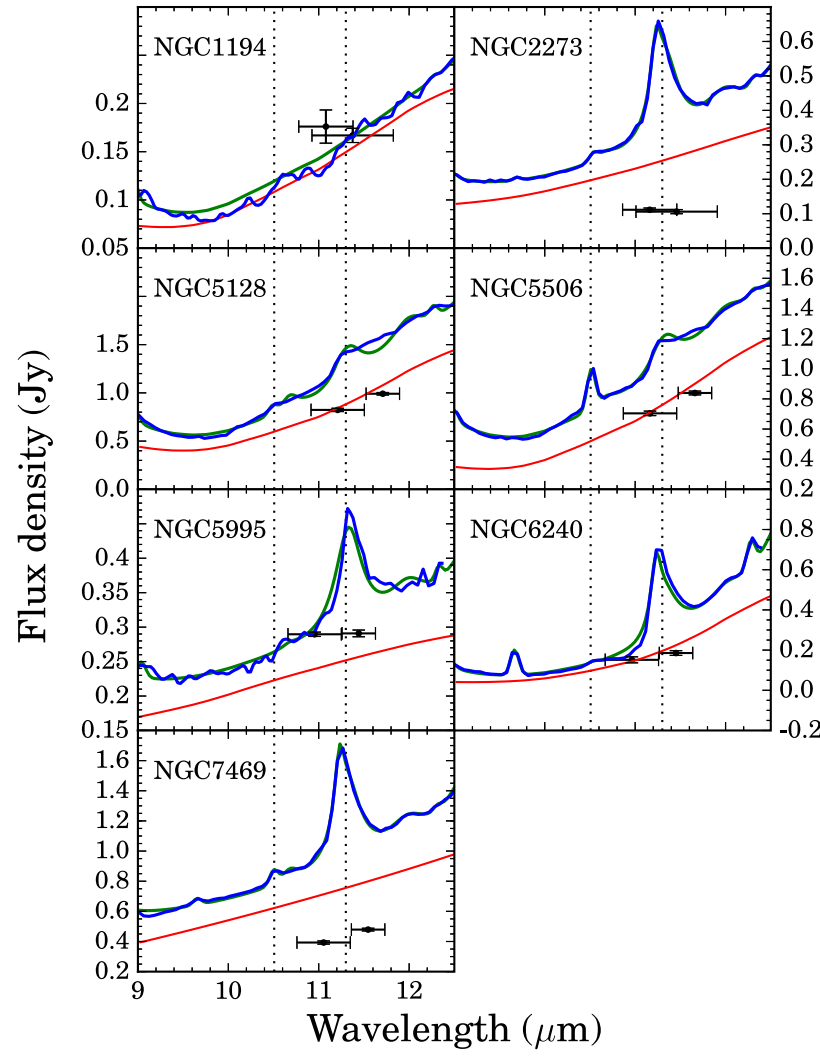

Figure 5. Same as Fig. 4 for galaxies 9-15.

features based solely on the photometric fluxes. We refer the reader to Appendix A for the technical details of this analysis.

Our results, presented in Table 2, show that seven out of the 15 galaxies in the sample have PAH emission. This number is different from the one presented in Section 3.1 due to the different approach. Most importantly, simulating the PAH emission band provides information on the effects of having some of the emissions sampled by the reference filter. Also, while in Section 3.1 the images were compared on a pixel by pixel basis, in the present analysis only the integrated photometry is considered, thus rendering PSF mismatch problems irrelevant. Therefore, not all galaxies identified in the image subtraction resulted in positive detections of PAH and vice versa.

Once known, the total flux emitted in the $11.3 \mu \mathrm{m}$ PAH flux can be used in conjunction with empirical relations for the SFR. Although this molecular feature has a less stringent correlation with SFR than the emission band at $8.6 \mu \mathrm{m}$ (Diamond-Stanic \& Rieke 2010), the features at $8.6 \mu \mathrm{m}$ appear to be observationally suppressed in the vicinity of the AGN, while the $11.3 \mu \mathrm{m}$ seems to be less affected (Alonso-Herrero et al. 2011). That apparent suppression should not be confused with the physical destruction of PAH molecules but rather the dilution of PAH emission in the intense continuum emission from the AGN (Sales, Pastoriza \& Riffel 2010; AlonsoHerrero et al. 2014).

As previously discussed in the Introduction, the $11.3 \mu \mathrm{m} \mathrm{PAH}$ band is a reliable MIR proxy for SFRs. It is particularly well suited for the study of AGN due to its relative insensitivity to the radiation from the accretion disc, which is thought to destroy the molecules (Voit 1992). This is not the case of atomic fine structure lines such as the $12.8 \mu \mathrm{m}$ [NeII], which tend to overestimate SFR in luminous 
AGNs (Diamond-Stanic \& Rieke 2010). Other PAH features on shorter wavelengths, such as the 6.2,7.7 and 8.6 $\mu \mathrm{m}$ features, have been shown to be suppressed in Seyferts (ibid.).

Based on templates of MIR spectra of starburst (SB) galaxies from Rieke et al. (2009), Diamond-Stanic \& Rieke (2012) derived the relation

$\dot{M}_{*}\left(\mathrm{M}_{\odot} \mathrm{yr}^{-1}\right)=9.6 \times 10^{-9} L_{\mathrm{PAH}}\left(\mathrm{L}_{\odot}\right)$

for SBs characterized by $\dot{M}_{*}<10 \mathrm{M}_{\odot} \mathrm{yr}^{-1}$, where $L_{\mathrm{PAH}}$ is the luminosity from the PAH $11.3 \mu \mathrm{m}$ band. This equation has been evaluated for galaxies with $10^{9.75}<L_{\mathrm{IR}}<10^{10.75}$, with a dispersion of 0.28 dex. Our sample has a median $L_{\mathrm{IR}}=10^{10.65}$ with three galaxies above $L_{\mathrm{IR}}=10^{11}$; therefore, we conservatively regard uncertainties in $\dot{M}_{*}$ as 1 dex.

Proceeding in this line of thought, the energy-mass equivalence allows the luminosity of the circumnuclear starburst $\left(L_{\mathrm{SB}}\right)$ to be expressed as function of the mass conversion rate from molecular gas to stars $\left(\dot{M}_{*}\right)$. Adding a scaling factor of 0.14 , Kawakatu \& Wada (2008) write $L_{\mathrm{SB}}$, at a particular instant, as

$L_{\mathrm{SB}}=0.14 \varepsilon \dot{M}_{*} c^{2}$,

where $c$ is the speed of light in the vacuum and $\varepsilon$ is the mass conversion efficiency set to 0.007 , which is the fraction of mass converted to energy during the fusion of hydrogen.

These last two equations, in cgs units, result in the following relation between PAH emission at $11.3 \mu \mathrm{m}$ and SB luminosities:

$L_{\mathrm{SB}}\left(\mathrm{erg} \mathrm{s}^{-1}\right)=\varepsilon c^{2} 2.2 \times 10^{-17} L_{\mathrm{PAH}}\left(\mathrm{erg} \mathrm{s}^{-1}\right)$.

SB luminosities, derived according to equation (4), for all the galaxies in the sample are presented in Table 2 .

It is important to point out that of all the seven galaxies in which we detected circumnuclear star formation, four have already been hinted at having such a characteristic by previous studies, namely ESO 138-G001, NGC 253, NGC 2273 and NGC 5506 (Mulchaey, Wilson \& Tsvetanov 1996; Engelbracht et al. 1998; Oliva et al. 1999; Cid Fernandes et al. 2005). Based on the assumption that MIR observations penetrate deeper into the dusty nuclear environment, one should expect such cases. In the opposite direction, NGC 6240 and NGC 7469 have previous detections of circumnuclear star formation in the optical, but we find no evidence for it in our analysis. This could be either due to the detection limit imposed here or, in the case of NGC 7469, because the star formation structure seen by previous authors is outside the pupil used to isolate the nuclear source (see Soifer et al. 2003).

The SFR estimates presented here for the circumnuclear region of some of the galaxies are considerably smaller than previous estimates for the entire galaxy found in the literature. In the case of NGC 6240, Howell et al. (2010) report an SFR of $148.44 \mathrm{M}_{\odot} \mathrm{yr}^{-1}$ using Spitzer $L_{\mathrm{IR}}$ and GALEX FUV measurements, with the caveat that some AGN contamination may be present. The most likely explanation for the smaller value of SFR presented here is the difference in the apertures, which is almost a factor of 10 smaller when compared to that of Spitzer.

On the other hand, NGC 253 and NGC 7469 have reported SFRs for the nuclear region with spatial resolutions comparable to the ones in this paper, with values of $2.8 \pm 0.3 \mathrm{M}_{\odot} \mathrm{yr}^{-1}$ and 2.6-5.1 $\mathrm{M}_{\odot} \mathrm{yr}^{-1}$, respectively (Ott et al. 2005; Davies et al. 2007). Our estimates for these targets are 1.1 and 0.6 dex smaller, therefore, justifying the conservative uncertainty of 1 dex mentioned above.

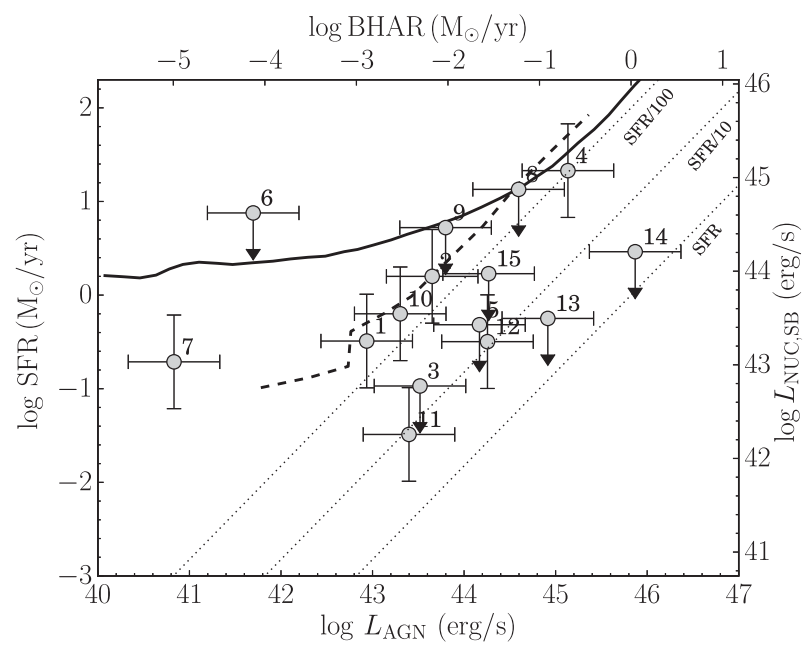

Figure 6. Relation between AGN luminosity and the nuclear SFR. The numbers correspond to the identification on the first column of Table 2. Dotted lines mark accretion rates in terms of the SFR, in ratios of BHAR = $\mathrm{SFR}, \mathrm{BHAR}=\mathrm{SFR} / 10$ and $\mathrm{BHAR}=\mathrm{SFR} / 100$. The solid line and dashed lines are reproductions of the semi-analytic models in Neistein \& Netzer (2014, see text).

\section{DISCUSSION}

A number of theoretical studies have shown that the interstellar environment in which SMBH accretion is observed also favours star formation in the circumnuclear region. For instance, Kawakatu \& Wada (2008) using a semi-analytic model show that given a continuous supply of gas from outer parts of the host galaxy to the inner $100 \mathrm{pc}$, AGN luminosity will be correlated to SB luminosity while the accretion rates are high. In another semi-analytic work, Neistein \& Netzer (2014) show that for SB and accretion events ignited by galaxy mergers, a correlation between $L_{\mathrm{AGN}}$ and SFR is verified when $L_{\mathrm{AGN}}>10^{42} \mathrm{erg} \mathrm{s}^{-1}$.

In order to investigate the possible physical connection between the AGN and the circumnuclear SB, we compared the SFRs probed by the PAH emission to the accretion rate of the AGNs. The latter quantity was derived from the X-ray $2-10 \mathrm{keV}$ luminosity, with bolometric corrections obtained from

$$
\begin{aligned}
& \log \left[\frac{L_{12}}{L(2-10 \mathrm{keV})}\right] \\
& =1.54+0.24 L_{12}+0.012 L_{12}^{2}-0.0015 L_{12}^{3}
\end{aligned}
$$

(Marconi et al. 2004), where $L_{12}=\log \left(L_{\mathrm{AGN}}\right)-12$ and $L_{\mathrm{AGN}}$ is the bolometric luminosity in units of $\mathrm{L}_{\odot}$. Numerical methods were used to solve this transcendental equation, resulting in correction factors roughly between 7 and 55. Resulting bolometric luminosities for all the AGNs in our sample are also shown in Table 2. We would like to emphasize that the value of $L_{\mathrm{AGN}}$ obtained from X-ray radiation is only representative of the instantaneous accretion rate of the BH. On the other hand, the emission from aromatic molecules used to assess SFRs lags $150 \mathrm{Myr}$ behind the main star formation event.

The points in Fig. 6 show the SFRs of circumnuclear regions versus the bolometric luminosities of the AGNs. In the same graph, we compare our results with theoretical predictions from Neistein \& Netzer (2014) by overplotting the average SFRs and $L_{\mathrm{AGN}}$ lines from Fig. 3 in the same paper. The solid line represents the average SFR for each $L_{\mathrm{AGN}}$ bin in Neistein \& Netzer 
(2014) models, while the dashed line marks the average $L_{\mathrm{AGN}}$ for each SFR bin. We also note that the higher luminosity AGNs in our sample $\left(L_{\mathrm{AGN}}>10^{42}\left(\mathrm{erg} \mathrm{s}^{-1}\right)\right)$ are mainly located in the region these authors claim to be occupied by objects in the early stages of the SB. Observational evidence from longer wavelengths also shows a similar trend for high-luminosity AGNs. For instance, Rosario et al. (2012), using 60- $\mu \mathrm{m}$ data from the Herschel space telescope, found that local AGN luminosities correlate with SFR for $L_{\mathrm{AGN}}>10^{44}\left(\mathrm{erg} \mathrm{s}^{-1}\right)$, thus agreeing with our findings.

Through the use of hydrodynamical simulations, Hopkins \& Quataert (2010) have predicted correlations between black hole accretion rates (BHARs) and SFR. They conclude that the SFRs are close to BHARs when considering only the nuclear region $(R<10 \mathrm{pc})$. The spread in the correlation increases as larger radii are considered, at the same time that BHARs correspond to smaller fractions of SFRs. In our analysis, we have not compensated the SFRs for the different projected areas they represent because the majority of our sources are unresolved. As a result, some of the dispersion in Fig. 6 can be directly linked to comparing different proportions of the host galaxy.

In Fig. 6, we also indicate the corresponding $L_{\mathrm{SB}}$ for the calculated SFRs, meaning the luminosity due exclusively to young stars. The most striking feature is that there are no galaxies where $L_{\mathrm{AGN}}>100 \times L_{\mathrm{SB}}$. In other words, all the AGN-harbouring galaxies in our sample, even the most energetic ones, have SBs radiating at least 1 per cent of the energy from the central engine. At the same time, the high-luminosity AGNs have circumnuclear SBs that at most match the energy output of the central source. Moreover, we find that two of the seven well-constrained galaxies lie within uncertainty limits of the one-to-one line, meaning that a significant fraction of these sources have nearly as much energy coming from the AGN as from the circumnuclear SB. This reinforces the importance of isolating the AGN emission from star formation at the MIR.

Concerning the low-luminosity AGNs, there is one galaxy that has more than 100 times more energy coming from young stars than from the AGN. This is in agreement with recent studies showing the huge importance of the circumnuclear star-forming components at all wavelengths to understand LLAGN (e.g. González-Martín et al. 2014). Conversely, we find no examples of low-luminosity AGNs with $L_{\mathrm{SB}} \ll L_{\mathrm{AGN}}$. Regarding the relationship between the luminosities from the different phenomena, our data agree with the theoretical predictions of Kawakatu \& Wada (2008) and Wutschik et al. (2013), which argue that low-luminosity AGNs should not display a correlation between circumnuclear star formation and AGN activity. According to these authors, this lack of correlation represents a stage where the circumnuclear disc has become gravitationally stable, thus ceasing the accretion by the $\mathrm{SMBH}$, but still has enough gas to form stars.

Diamond-Stanic \& Rieke (2012) have found a positive correlation between BHAR and the SFR in radii averaging to $300 \mathrm{pc}$, throughout their sample, using the [OIV] as an indicator of the former. In that study, only two of the four points with $L_{\mathrm{AGN}}<42 \mathrm{erg} \mathrm{s}^{-1}$ fall within the correlation, leaving further two points below it. This result contrasts with our analysis in the sense that we would expect to see higher circumnuclear star formation in the low-luminosity regime. Using spectra rather than images, Esquej et al. (2014) arrived at circumnuclear star formation detection rates similar to ours, almost half of their sample of 23 AGNs. We have four objects in common with their work, and our estimates for $11.3 \mu \mathrm{m}$ PAH luminosity agree with theirs within the uncertainties.

\section{CONCLUSIONS}

We analysed MIR images of a sample of 15 AGNs in two adjacent filters (PAH2 and Si5 in the CanariCam, and PAH2 and PAH2_2 at VISIR) to study the occurrence of circumnuclear star formation via the $11.3 \mu \mathrm{m}$ PAH emission band. Three of these 15 galaxies were observed with GTC/CanariCam, and the remaining 12 with ESO/VISIR, with images taken from the atlas published by Asmus et al. (2014). We have also presented new high-resolution MIR images for NGC 2146, which was eventually left out of the remaining analysis due to the lack of a clearly detectable nucleus. Our main results are as follows.

(i) Circumnuclear star formation, at distances as low as $20 \mathrm{pc}$ from the nucleus, was detected in seven out of the 15 galaxies. Among these seven galaxies, four show unresolved emission, NGC 253 has clearly identifiable star formation nodes and ESO 005-G004 and NGC 2273 have not been detected in the image subtraction.

(ii) The luminosity from the circumnuclear SB correlates with the bolometric luminosity of the central engine only for AGNs with luminosity higher than $10^{42} \mathrm{erg} \mathrm{s}^{-1}$. In the lower luminosity regime, we find that the radiative energy output due to star formation tends to be higher than the central engine.

\section{ACKNOWLEDGEMENTS}

DRD thanks Conselho Nacional de Desenvolvimento Científico e Tecnológico (CNPq) and Coordenação de Aperfeiçoamento de Pessoal de Nível Superior (CAPES) for partially supporting this research through fellowships. RR thanks CNPq and CAPES for finnancial support. This research is partially based on observations made with the GTC, installed at the Spanish Observatorio del Roque de los Muchachos of the Instituto de Astrofísica de Canarias, in the island of La Palma. This research has been partially supported by the Spanish Ministry of Economy and Competitiveness (MINECO) under the grant (project ref. AYA 2012 -39168-C03-01). OGM acknowledges the Juan de la Cierva fellowship.

\section{REFERENCES}

Alonso-Herrero A. et al., 2011, ApJ, 736, 82

Alonso-Herrero A. et al., 2014, MNRAS, 443, 2766

Asmus D., Honig S. F., Gandhi P., Smette A., Duschl W. J., 2014, MNRAS, 439, 1648

Awaki H., Terashima Y., Higaki Y., Fukazawa Y., 2009, PASJ, 61, S317

Bianchi S., Guainazzi M., Matt G., Fonseca Bonilla N., Ponti G., 2009, A\&A, 495, 421

Cid Fernandes R., Heckman T., Schmitt H., Delgado R. M. G., StorchiBergmann T., 2001, ApJ, 558, 81

Cid Fernandes R., Mateus A., Sodré L., Stasińska G., Gomes J. M., 2005, MNRAS, 358, 363

Corral A., Della Ceca R., Caccianiga A., Severgnini P., Brunner H., Carrera F. J., Page M. J., Schwope A. D., 2011, A\&A, 530, A42

Davies R. I., Mueller Sanchez F., Genzel R., Tacconi L. J., Hicks E. K. S., Friedrich S., Sternberg A., 2007, ApJ, 671, 1388

Diamond-Stanic A. M., Rieke G. H., 2010, ApJ, 724, 140

Diamond-Stanic A. M., Rieke G. H., 2012, ApJ, 746, 168

Díaz-Santos T., Alonso-Herrero A., Colina L., Packham C., Levenson N. A., Pereira-Santaella M., Roche P. F., Telesco C. M., 2010, ApJ, 711, 328

Engelbracht C. W., Rieke M. J., Rieke G. H., Kelly D. M., Achtermann J. M., 1998, ApJ, 505, 639

Esquej P. et al., 2014, ApJ, 780, 86

Ferrarese L., Merritt D., 2000, ApJ, 539, L9

Filippenko A. V., Halpern J. P., 1984, ApJ, 285, 458 
Forbes D. A., Polehampton E., Stevens I. R., Brodie J. P., Ward M. J., 2000, MNRAS, 312, 689

Gallimore J. F. et al., 2010, ApJS, 187, 172

Gebhardt K. et al., 2000, ApJ, 539, L13

González-Delgado R. M., Pérez E., 1993, Ap\&SS, 205, 127

González-Martín O., Vaughan S., 2012, A\&A, 544, A80

González-Martín O., Masegosa J., Márquez I., Guerrero M. A., DultzinHacyan D., 2006, A\&A, 460, 45

González-Martín O. et al., 2013, A\&A, 553, A35

González-Martín O., Díaz-González D., Acosta-Pulido J. A., Masegosa J., Papadakis I. E., Rodríguez-Espinosa J. M., Márquez I., HernándezGarcía L., 2014, A\&A, 567, A92

Greenhill L. J., Tilak A., Madejski G., 2008, ApJ, 686, L13

Guainazzi M., Fabian A. C., Iwasawa K., Matt G., Fiore F., 2005, MNRAS, 356,295

Gültekin K. et al., 2009, ApJ, 698, 198

Hao L. et al., 2005, ApJ, 625, L75

Hopkins P. F., Quataert E., 2010, MNRAS, 407, 1529

Howell J. H. et al., 2010, ApJ, 715, 572

Kauffmann G. et al., 2003, MNRAS, 346, 1055

Kawakatu N., Wada K., 2008, ApJ, 681, 73

Kormendy J., Richstone D., 1995, ARA\&A, 33, 581

Lira P., Johnson R. A., Lawrence A., Cid Fernandes R., 2007, MNRAS, 382, 1552

McConnell N. J., Ma C.-P., 2013, ApJ, 764, 184

Marconi A., Risaliti G., Gilli R., Hunt L. K., Maiolino R., Salvati M., 2004, MNRAS, 351, 169

Marinucci A., Bianchi S., Nicastro F., Matt G., Goulding A. D., 2012, ApJ, 748,130

Martini P., Regan M. W., Mulchaey J. S., Pogge R. W., 2003, ApJS, 146, 353

Mason R. E. et al., 2012, AJ, 144, 11

Mulchaey J. S., Wilson A. S., Tsvetanov Z., 1996, ApJS, 102, 309

Müller-Sánchez F., González-Martín O., Fernández-Ontiveros J. A., AcostaPulido J. A., Prieto M. A., 2010, ApJ, 716, 1166

Nandra K., O’Neill P. M., George I. M., Reeves J. N., 2007, MNRAS, 382, 194

Neistein E., Netzer H., 2014, MNRAS, 437, 3373

Nenkova M., Sirocky M. M., Ivezić v., Elitzur M., 2008, ApJ, 685, 147

Oliva E., Origlia L., Maiolino R., Moorwood A. F. M., 1999, A\&A, 350, 9

Ott J., Weiss A., Henkel C., Walter F., 2005, ApJ, 629, 767

Piconcelli E., Bianchi S., Vignali C., Jiménez-Bailón E., Fiore F., 2011, A\&A, 534, A126

Ramos Almeida C. et al., 2011, MNRAS, 417, L46

Rieke G. H., Alonso-Herrero A., Weiner B. J., Pérez-González P. G., Blaylock M., Donley J. L., Marcillac D., 2009, ApJ, 692, 556

Riffel R., Pastoriza M. G., Rodríguez-Ardila A., Maraston C., 2007, ApJ, 659, L103

Rodriguez Espinosa J. M., Rudy R. J., Jones B., 1987, ApJ, 312, 555

Rosario D. et al., 2012, A\&A, 545, A45

Ruschel-Dutra D., Pastoriza M., Riffel R., Sales D. A., Winge C., 2014, MNRAS, 438, 3434

Rush B., Malkan M. A., Spinoglio L., 1993, ApJS, 89, 1

Sales D. A., Pastoriza M. G., Riffel R., 2010, ApJ, 725, 605

Sales D. A., Pastoriza M. G., Riffel R., Winge C., Rodríguez-Ardila A., Carciofi A. C., 2011, ApJ, 738, 109

Sales D. A., Ruschel-Dutra D., Pastoriza M. G., Riffel R., Winge C., 2014, MNRAS, 441, 630

Shinozaki K., Miyaji T., Ishisaki Y., Ueda Y., Ogasaka Y., 2006, AJ, 131, 2843

Shu X. W., Yaqoob T., Wang J. X., 2011, ApJ, 738, 147

Siebenmorgen R., Krügel E., Spoon H. W. W., 2004, A\&A, 414, 123

Silk J., Rees M. J., 1998, A\&A, 331, L1

Smith J. D. T. et al., 2007, ApJ, 656, 770

Soifer B. T., Bock J. J., Marsh K., Neugebauer G., Matthews K., Egami E., Armus L., 2003, AJ, 126, 143

Stasińska G., Asari N. V., Fernandes R. C., Gomes J. M., Schlickmann M., Mateus A., Schoenell W., Sodré L., Jr, 2008, MNRAS, 391
Sturm E. et al., 2005, ApJ, 629, L21

Thompson G. D., Levenson N. A., Uddin S. A., Sirocky M. M., 2009, ApJ, 697,182

Tielens A., 2008, ARA\&A, 46, 289

Ueda Y., Ishisaki Y., Takahashi T., Makishima K., Ohashi T., 2005, ApJS, 161,185

Ueda Y. et al., 2011, PASJ, 63, S937

Véron-Cetty M.-P., Véron P., 2010, A\&A, 518, A10

Voit G. M., 1992, MNRAS, 258, 841

Vollmer B., Davies R. I., 2013, A\&A, 556, A31

Winter L. M., Mushotzky R. F., Reynolds C. S., Tueller J., 2009, ApJ, 690, 1322

Wu Y., Charmandaris V., Huang J., Spinoglio L., Tommasin S., 2009, ApJ, 701,658

Wutschik S., Schleicher D. R. G., Palmer T. S., 2013, A\&A, 560, A34

\section{APPENDIX A: ESTIMATING TOTAL INTENSITY OF THE PAH BAND}

In this section, we will discuss the central issue concerning the determination of SFR with the available data, which mainly consists of disentangling PAH and continuum emission with two overlapping filters. Let us assume that the specific portion of the spectrum in which we are interested, can be well represented by a continuum plus the PAH emission band

$S(\lambda)=C(\lambda)+I(\lambda)$.

The latter is described here by the sum of two Drude profiles parametrized by their central intensities as in Smith et al. (2007):

$I_{v}=\frac{b_{r} \gamma_{r}}{\left(\lambda / \lambda_{r}-\lambda_{r} / \lambda\right)^{2}+\gamma_{r}^{2}}$,

where $b_{r}$ is the central intensity, $\gamma_{r}$ is the fractional FWHM $/ \lambda_{r}$ and $\lambda_{r}$ is the central wavelength (Smith et al. 2007). The values of $\lambda_{r}$ and $\gamma_{r}$ that define the first component are $11.22 \mu \mathrm{m}$ and 0.012 , respectively, while $11.33 \mu \mathrm{m}$ and 0.032 represent the second component. Just as in Section 3.1, the spectral decomposition code PAHFIT (Smith et al. 2007) was employed to obtain individual models for the continuum of each galaxy based on Spitzer/IRS spectra. Fig. A1 shows an example of a modelled spectrum, with the average continuum of our sample. In this example, and in all subsequent analyses, the central intensity of both Drude profiles was kept equal. Tests were performed to evaluate the impact of different ratios of central intensities, and no large differences were found.

The total flux $f_{n}$ measured in each filter is essentially a weighted average of the spectrum, with the weight for each wavelength given by the filter's transmission function. Thus,

$f_{n}=\int F_{\mathrm{n}}(\lambda) S(\lambda) \mathrm{d} \lambda$,

where $F_{\mathrm{n}}$ is the normalized transmission curve. It is easy to see that, within the framework of this model, the difference in flux between filters, that is $f_{\mathrm{PAH}}-f_{\mathrm{REF}}$, can be written as function of only the central intensity of the PAH emission profiles, and the redshift of the galaxy:

$$
\begin{aligned}
D\left(b_{r}, z\right)= & \int_{\lambda_{0}}^{\lambda_{1}}\left[C_{\nu}(\lambda, z)+I_{v}\left(b_{r}, \lambda, z\right)\right] \\
& \times\left[F_{1}(\lambda)-F_{2}(\lambda)\right] \mathrm{d} \lambda
\end{aligned}
$$

where $C_{v}(\lambda, z)$ is the continuum emission, $I_{v}\left(b_{r}, \lambda, z\right)$ is the PAH emission in the $11.3 \mu \mathrm{m}$ band and $F_{1}(\lambda)$ and $F_{2}(\lambda)$ are the normalized transmission functions for the PAH and reference filters, respectively. The total intensity of the PAH band is given by the 


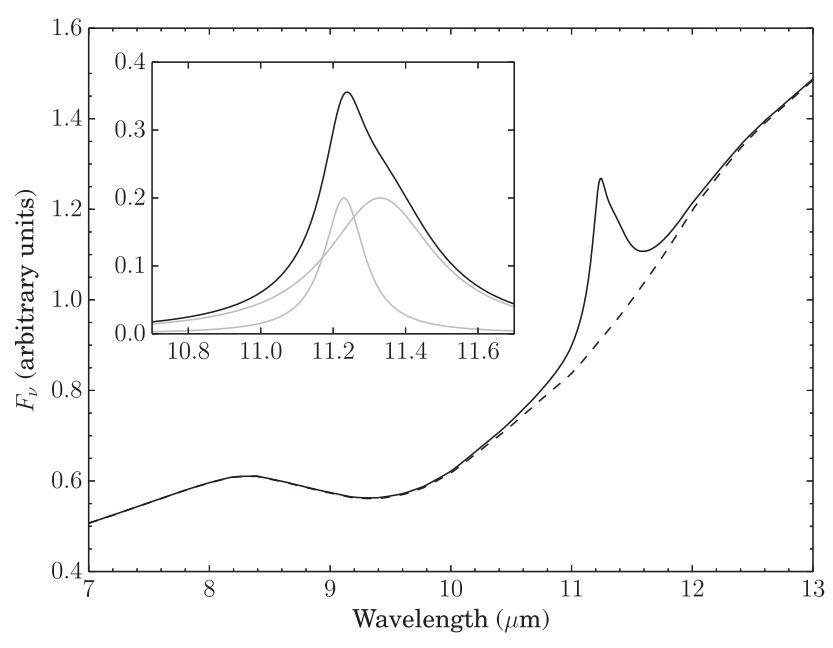

Figure A1. Example of simulated spectrum used to study the effects of redshift and filter subtraction on the estimate of PAH emission. The spectrum that would be observed (solid black line) is the result of the sum of a continuum emission absorbed by silicate grains (dashed line) and the emission from PAH molecules. The smaller plot window highlights the composition of the $11.3 \mu \mathrm{m}$ by two distinct Drude profiles (grey lines.)

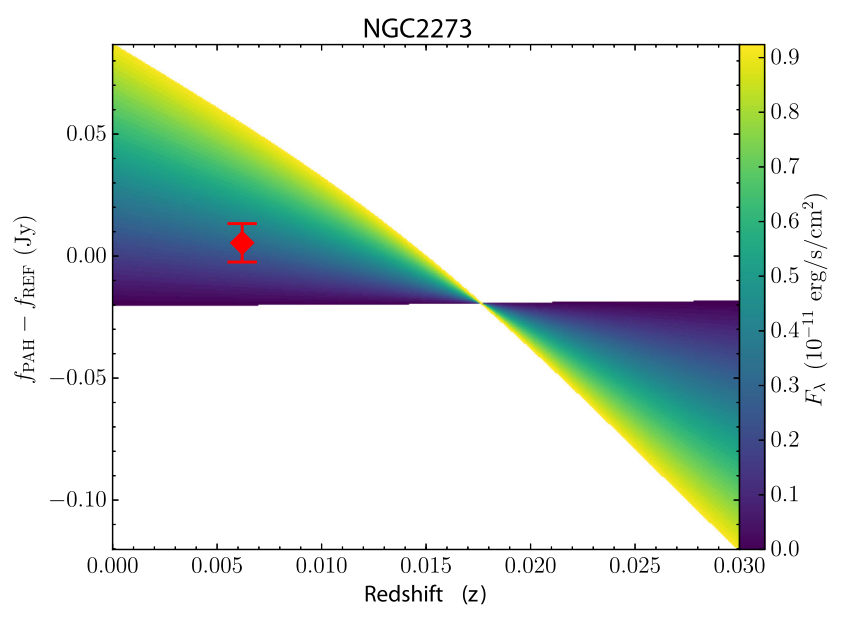

Figure A2. Analysis of the effects of redshift and total intensity on the difference between the filters on CanariCam. At a redshift of 0.017, the reference filter begins to have a higher flux than the emission filter even for the highest intensities allowed in the models.

sum of the total intensity of each component, which in turn is the integral of equation (A2):

$F_{\mathrm{PAH}}=\frac{\pi c}{2}\left(\frac{b_{1} \gamma_{1}}{\lambda_{1}}+\frac{b_{2} \gamma_{2}}{\lambda_{2}}\right)$,

where the sub-indexes represent the different components. Therefore, once equation (A4) is solved for a particular central intensity $b_{r}$, and assuming that the redshift $z$ is known, we can use the result in equation (A5) to calculate the total flux in the $11.3 \mu \mathrm{m}$ PAH band. Numerical methods were employed to investigate the solutions of equation (A4), and Figs A2-A4 show three distinct examples of typical outcomes.

In Fig. A2, we show a map of solutions in the plane $f_{\mathrm{PAH}}-f_{\mathrm{REF}}$, which we assume to be equal to $D\left(b_{r}, z\right)$ versus $z$, for the inferred continuum of NGC 2273 and the CanariCam filters. The red diamond in this figure represents the measured filter difference,

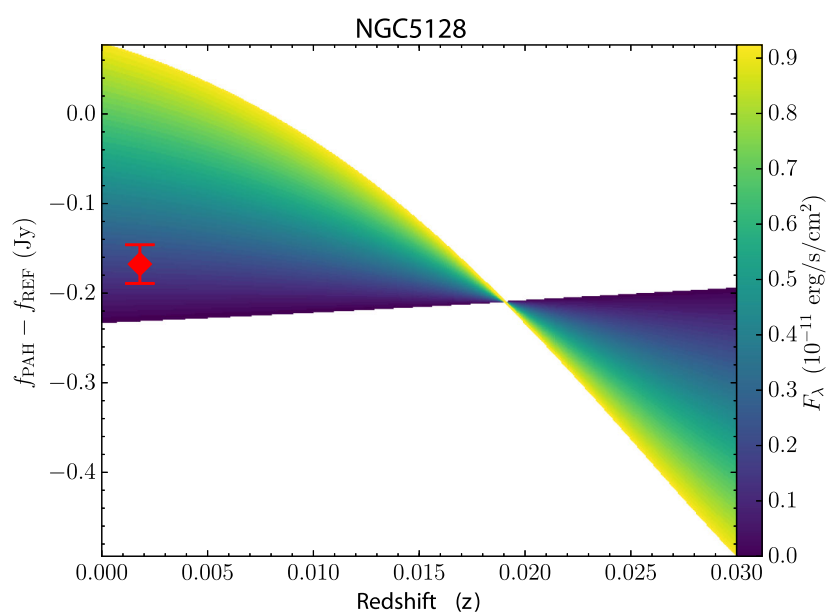

Figure A3. Same as Fig. A2 for the galaxy NGC 5128.

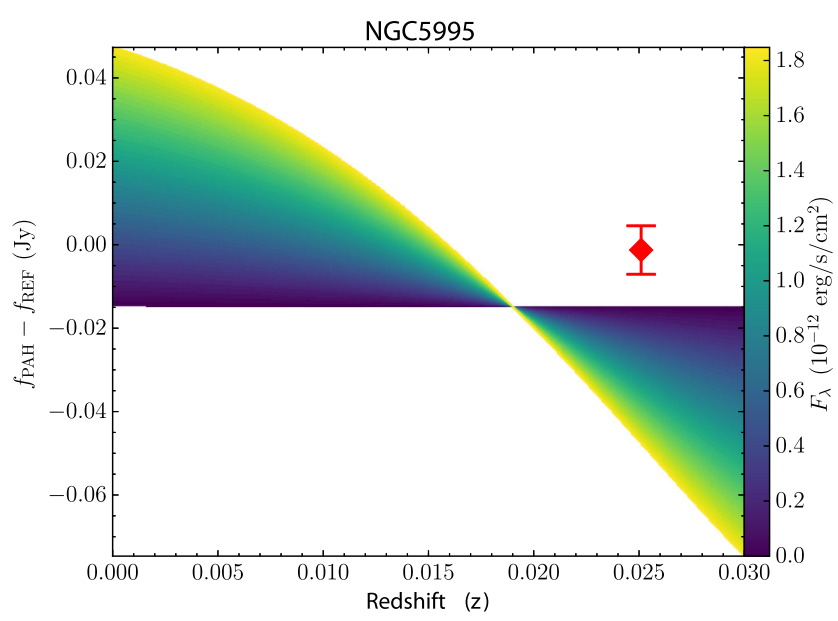

Figure A4. Same as Fig. A2 for the galaxy NGC 5995.

along with $1 \sigma$ error bars. The colour scale represents the total flux of the simulated PAH band, from deep blue for no emission to bright green for the maximum simulated emission. The latter is completely arbitrary and was chosen for readability purposes. In the case of NGC 2273, the almost flat slope of the continuum has no clear impact on the difference between fluxes for any of the sampled redshift coordinates. Nevertheless, it is already clear that even a modestly negative value of $D$ is still compatible with a PAH emission of about $0.2 \mathrm{erg} \mathrm{s}^{-1} \mathrm{~cm}^{-2}$. At a redshift of 0.017 , the situation is reversed, because the reference filter $\left(f_{\mathrm{REF}}\right)$ begins to sample more of the PAH band than the original PAH filter. Thus, for the same continuum shape and for the same filters, the PAH emission of a galaxy with redshift $z>0.017$ would cause $f_{\mathrm{PAH}}-f_{\mathrm{REF}}$ to be lower than $-0.2 \mathrm{Jy}$.

Fig. A3 shows the example of NGC 5128, which has a notably steeper continuum (see Fig. 5). At the redshift of this galaxy, the flux of PAH band would have to be almost $8 \times 10^{-10} \mathrm{erg} \mathrm{s}^{-1} \mathrm{~cm}^{-2}$ for the difference between fluxes to be zero. The negative flux seen in the image subtraction of Section 3.1 is therefore completely compatible with PAH emission, although the former alone is not enough to warrant an emission detection. The slow rise in the minimum value of $f_{\mathrm{PAH}}-f_{\mathrm{REF}}$ that can be seen in Fig. A3 is caused by the change in continuum slope as one moves towards longer wavelengths, leaving the silicate absorption band at $9.7 \mu \mathrm{m}$. 
Finally, we discuss the case of the NGC 5995, the galaxy with the highest redshift in our sample. This galaxy is well beyond the threshold where the reference filter has more of the PAH band than the original PAH filter, which in the case of VISIR happens at $z=0.019$. A positive detection would therefore be characterized by $f_{\mathrm{PAH}}-f_{\mathrm{REF}} \leq-0.015 \mathrm{Jy}$. However, the photometric measurements show practically no difference between the fluxes in both filters, thus placing NGC 5995 in a region which would imply a negative PAH flux, or a more likely misrepresentation of the underlying continuum. We classify such cases as non-detections and report PAH fluxes and derived quantities as upper limits, based on the photometric uncertainty. As for the reasons that might have thus affected the continuum, the most probable is a simple overestimation of its declivity. Another possibility would be the presence of silicate emission at the $9.7 \mu \mathrm{m}$ band, rather than the more usual absorption. These emission features are a common occurrence among QSOs (e.g. Hao et al. 2005) and have also been identified in many local AGNs (Sturm et al. 2005; Thompson et al. 2009; Mason et al. 2012; Ruschel-Dutra et al. 2014) and are predicted by radiative transfer models based on clumpy tori even for Seyfert 2s (Nenkova et al. 2008).

This paper has been typeset from a $\mathrm{T}_{\mathrm{E}} \mathrm{X} / \mathrm{LT} \mathrm{E} \mathrm{X}$ file prepared by the author. 